\title{
Identification of protein tyrosine phosphatase SHP-2 as a new target of perfluoroalkyl acids in HepG2 cells
}

\author{
Yu Yang ${ }^{1}$ Qi-Yan $\mathrm{Lv}^{1} \cdot$ Liang-Hong Guo ${ }^{1,2} \cdot$ Bin Wan $^{1} \cdot$ Xiao-Min Ren $^{1} \cdot$ Ya-Li Shi $^{1}$ • \\ Ya-Qi Cai ${ }^{1}$
}

Received: 5 April 2016 / Accepted: 24 August 2016 / Published online: 29 August 2016

(C) Springer-Verlag Berlin Heidelberg 2016

\begin{abstract}
Perfluoroalkyl acids (PFAAs) are widespread environmental contaminants which have been detected in humans and linked to adverse health effects. Previous toxicological studies mostly focused on nuclear receptormediated pathways and did not support the observed toxic effects. In this study, we aimed to investigate the molecular mechanisms of PFAA toxicities by identifying their biological targets in cells. Using a novel electrochemical biosensor, 16 PFAAs were evaluated for inhibition of protein tyrosine phosphatase SHP-2 activity. Their potency increased with PFAA chain length, with perfluorooctadecanoic acid (PFODA) showing the strongest inhibition. Three selected PFAAs, $25 \mu \mathrm{M}$ perfluorooctanoic acid (PFOA), perfluorooctane sulfonic acid, and PFODA, also inhibited SHP-2 activity in HepG2 cells and increased paxillin phosphorylation level. PFOA was detected in the immunoprecipitated SHP-2 from the cells exposed to $250 \mu \mathrm{M}$ PFOA, providing unequivocal evidence for the direct binding of PFOA with SHP-2 in the cell. Molecular docking rationalized the formation of PFAA/SHP-2
\end{abstract}

Yu Yang and Qi-Yan Lv contributed equally to this work.

Electronic supplementary material The online version of this article (doi:10.1007/s00204-016-1836-2) contains supplementary material, which is available to authorized users.

Liang-Hong Guo

LHGuo@rcees.ac.cn

1 State Key Laboratory of Environmental Chemistry and Ecotoxicology, Research Center for Eco-Environmental Sciences, Chinese Academy of Sciences, 18 Shuangqing Road, P.O. Box 2871, Beijing 100085, China

2 Institute of Environment and Health, Jianghan University, Wuhan 430056, China complex and chain length-dependent inhibition potency. Our results have established SHP-2 as a new cellular target of PFAAs.

Keywords Perfluoroalkyl acids · Protein tyrosine phosphatase $\cdot$ Inhibition $\cdot$ Cellular target

\section{Introduction}

Perfluoroalkyl acids (PFAAs), including perfluoroalkyl carboxylic acids (PFCAs) and perfluoroalkyl sulfonic acids (PFSAs; Supplementary Material Fig. S1), are an emerging class of environmental contaminants. They are widely used in industrial and commercial products, and frequently detected in various environmental media (Giesy and Kannan 2002). Due to their extremely high chemical stability, PFAAs hardly degrade in the environment or organisms, and therefore persist and bioaccumulate over long duration. A number of bio-monitoring investigations have detected PFAAs in humans in many regions of the world, but with considerable variation among populations as far as their level of exposure is concerned (Perez et al. 2013). The highest blood concentration was found among workers in PFAA-producing plants, with mean concentrations of $1000-2000 \mathrm{ng} / \mathrm{mL}$ for perfluorooctane sulfonate (PFOS) and $5000 \mathrm{ng} / \mathrm{mL}$ for perfluorooctanoic acid (PFOA), whereas general populations on average had concentrations (ng/mL) of 35 (PFOS), 5 (PFOA), 2 (perfluorohexanesulfonate, PFHS), and 1.5 (perfluorononanoic acid, PFNA) (Conder et al. 2008; Ng and Hungerbuhler 2014; Kato et al. 2011). Long elimination half-lives have been observed for some PFAAs in humans, for example, means of 3.8 years for PFOA, 5.4 years for PFOS and 8.5 years for PFHS (Olsen et al. 2007). 
High human exposure levels of PFAAs have raised concerns about their adverse health effects. Epidemiological studies revealed positive correlations between PFAA concentrations in human and some diseases such as cancers (kidney, prostate, testicular, ovarian, and thyroid cancer) (Chang et al. 2014; Eriksen et al. 2009; Barry et al. 2013; Vieira et al. 2013), asthma (Humblet et al. 2014), and cardiovascular diseases (Simpson et al. 2013), as well as dysfunctions of reproductive and thyroid systems (Toft et al. 2012; Kristensen et al. 2013; Wang et al. 2014a, b). In view of the potential health risks, toxicological studies of PFAAs were carried out extensively on experimental animals and cells. In rats, PFOS and PFOA demonstrated modest acute toxicity, with average $\mathrm{LD}_{50}$ values of 250 and $540 \mathrm{mg} / \mathrm{kg} /$ day, respectively (Sato et al. 2009; Griffith and Long 1980). In subacute tests, liver and thyroid are the most sensitive target organs of mice and rats. Other effects include reproductive and developmental toxicity (White et al. 2011a, b), neurotoxicity, immunotoxicity (DeWitt et al. 2009), and endocrine disruption (White et al. 2011a, b). In chronic tests, hepatotoxicity and carcinogenicity were observed in the rats exposed to PFOS (Wang et al. 2015). However, PFAAs did not show any genotoxicity in various in vitro and in vivo assays (Florentin et al. 2011), suggesting that the carcinogenic effects observed in rats probably result from mechanisms not directly acting on genes.

Observed hepatotoxic effects include hepatomegaly, reduced triglyceride and cholesterol levels, and increased expression of enzymes involved in $\beta$-oxidation and fatty acid $\omega$-oxidation (Zhang et al. 2008; Kudo et al. 2006). All these are biomarkers of exposure to peroxisome proliferators (PPs). PPs are known to induce carcinogenesis by mechanisms including prolonged oxidative stress and uncontrolled cell replication. Because these biological responses are mediated by peroxisome proliferator-activated receptor $\alpha(\operatorname{PPAR} \alpha)$, a ligand-activated transcription factor expressed primarily in the liver, early studies on the mode of action of PFAAs focused almost exclusively on this mechanism. Indeed, PFOS and PFOA were found to bind to the PPAR $\alpha$ ligand-binding domain and activate the receptor in reporter gene assays. However, PFAAs are only weak agonists when compared with PPAR $\alpha$ natural ligands such as long-chain unsaturated fatty acids (VandenHeuvel et al. 2006). In addition, exposure of PPAR $\alpha$ knockout mice to PFOA also induced hepatomegaly (Yang et al. 2002). These results suggest that PFAA hepatotoxicity cannot be attributed solely to PPAR $\alpha$-mediated pathways. Extrapolation of this mechanism to human is further hindered by the fact that hepatic expression of the receptor in human is much lower than in mice and rats (Wolf et al. 2012). More recent studies have investigated other nuclear receptors and enzymes as possible biological targets of PFAAs, such as estrogen receptor (Benninghoff et al. 2011), PPAR $\gamma$ (Zhang et al. 2013a, b), thyroid hormone receptor (Ren et al. 2015), and lysine decarboxylase (Wang et al. 2014a, b).

Protein phosphorylation is a common regulatory process involved in many intracellular signaling pathways. These pathways are dependent on the coordinated actions of protein kinases and protein phosphatases to regulate protein phosphorylation level. SHP-2 is a ubiquitously expressed cytoplasmic protein tyrosine phosphatase. It mediates cell signaling by growth factors and cytokines, and the activation of mitogen-activated protein (MAP) kinases Erk1/2 in particular (Deb et al. 1998). PTPN11 (encoding SHP-2) mutations have been found in Noonan syndrome, juvenile myelomonocytic leukemia, and several types of human malignancies (Tartaglia et al. 2001; Muller et al. 2013; Mohi and Neel 2007; Tartaglia et al. 2004). The direct association between SHP-2 hyperactivation and human diseases has stimulated tremendous interest in targeting the protein for therapeutic treatment, and a few SHP-2 inhibitors have been discovered (Chen et al. 2006; Liu et al. 2011; Yu et al. 2013; Butterworth et al. 2014). However, recent studies have revealed SHP-2 as a tumor suppressor. Selective deletion of SHP-2 leads to the development of tumors in the liver of aged mice (Bard-Chapeau et al. 2011), and promoted melanoma progression and metastasis in CD4+ T cells (Zhang et al. 2013a, b). In addition, decreased SHP-2 expression was observed in a small fraction of human hepatocellular carcinoma patient samples (Jiang et al. 2012). It was suggested that the dual functions of tumor promotion and tumor suppression depended on cellular contexts ( $\mathrm{Li}$ et al. 2012).

Considering the observed hepatotoxicity of PFAAs, insufficient knowledge on their mode of action, and important roles of SHP-2 implicated in liver tumors, we sought to find out whether SHP-2 would be a potential target of PFAAs in liver cells. We first screened 16 PFAAs for their inhibitory effect on SHP-2 using an electrochemical biosensor-based enzyme activity assay. We then confirmed the inhibition reaction and subsequent effect on protein phosphorylation in HepG2 cells after exposure to PFAAs. To prove that the biological effects in cells are the results of direct interaction of PFAAs with SHP-2, we captured the protein in cells by immunoprecipitation and detected the PFAA associated with the protein.

\section{Materials and methods}

\section{Electrochemical measurement of SHP-2 activity and inhibition by PFAAs}

In the sensor, a tyrosine-containing polypeptide, poly(glu, tyr) (4:1) peptide, was used as the enzyme substrate, and was covalently immobilized on the surface of ITO 
electrode by silane chemistry (Yang et al. 2011). Electroactive tyrosine residue, as the only receptor of phosphorylation and dephosphorylation in polypeptide, was used as a signal reporter. Tyrosine could be electrocatalytically oxidized with an osmium polypyridyl complex leading to the enhanced oxidation current, but the phosphorylation of tyrosine residue caused suppression of the oxidation current response (Qu et al. 2008). The distinct electrochemical behavior of tyrosine and phosphotyrosine enabled us to determine whether phosphorylation and dephosphorylation were achieved on a polypeptide or not. When EGFRcatalyzed tyrosine phosphorylation, and subsequent SHP2-catalyzed dephosphorylation were achieved, the tyrosine residue in polypeptide gave a high current response (Supplementary Material Fig. S2A,B). When the dephosphorylation was successfully inhibited, the tyrosine oxidation response was suppressed. The instrument measures the voltammetric oxidation current of tyrosine catalyzed by a dissolved electron mediator Os(bpy) ${ }_{3}^{2+}$ (bpy $=2,2^{\prime}$-bipyridine) (Hamann et al. 2005) to assess SHP-2 activity and its inhibition by PFAAs. SHP-2 activity assay was performed according to the following protocol. Firstly, the phosphorylation reaction mixture containing $25 \mathrm{mM}$ HEPES ( $\mathrm{pH} 7.4$ ), $2 \mathrm{mM} \mathrm{MnCl}_{2}, 5 \mathrm{mM} \mathrm{MgCl}_{2}, 50 \mu \mathrm{M}$ ATP, and $150 \mathrm{UmL}^{-1}$ EGFR in a total volume of $10 \mu \mathrm{L}$ was deposited onto the surface of the polypeptide-modified electrode and incubated in a moisturized container at $30{ }^{\circ} \mathrm{C}$ for $35 \mathrm{~min}$. Then, the dephosphorylation reaction on ITO electrode was performed in $10 \mu \mathrm{L}$ phosphate buffer, containing $50 \mathrm{mM}$ $\mathrm{NaCl}, 1 \mathrm{mM}$ DTT, $0.05 \%$ Tween-20, and SHP-2 and incubated in a moisturized container at $30{ }^{\circ} \mathrm{C}$ for $45 \mathrm{~min}$ (Supplementary Material Fig. S3). After each surface reaction, the electrodes were washed for 5 min each in $20 \mathrm{mM} \mathrm{PB}$ (with $0.1 \%$ Tween-20), $20 \mathrm{mM}$ PB (with $150 \mathrm{mM} \mathrm{NaCl}$ ), and $20 \mathrm{mM}$ PB on a shaker and then dried with nitrogen. As for the inhibition experiments, varying concentrations of inhibitors were included in the reaction mixture. The time-dependent inhibition of SHP-2 by PFAAs was investigated (Supplementary Material Fig. S4). After washing in $\mathrm{PB}$, cyclic voltammograms $(\mathrm{CVs})$ were measured in $3 \mu \mathrm{M}$ Os(bpy) ${ }_{3}^{2+} / 20 \mathrm{mM}$ PB (pH 7.4). From the dose-effect curve, an $\mathrm{IC}_{50}$ value (the concentration of the inhibitor required to reduce signal by $50 \%$ ) was obtained. Electrochemical measurements were taken on a CHI 660B electrochemistry analyzer from $\mathrm{CH}$ Instruments (Austin, TX) with an indium tin oxide (ITO) work electrode, a Pt counter electrode, and an $\mathrm{Ag} / \mathrm{AgCl}$ reference electrode.

\section{SHP-2 activity assay and total SHP-2 measurement}

For SHP-2 activity analysis, the cell lysates were collected in specific lysis buffer and divided into two parts, each for
SHP-2 activity assay and ELISA determination of SHP-2 protein. The SHP-2 activity of each sample was measured using the Human/Mouse/Rat Active SHP-2 Kit. In brief, SHP-2 immunoprecipitate was prepared from cell lysate using anti-SHP-2 antibody-conjugated agarose immunoprecipitation (IP) beads for $3 \mathrm{~h}$ at $4{ }^{\circ} \mathrm{C}$. After washing away unbound lysate, a synthetic phosphopeptide substrate was added and the reaction mixture was incubated for $30 \mathrm{~min}$ at $37{ }^{\circ} \mathrm{C}$ with continuous shaking at $700 \mathrm{rpm}$. Thereafter, $50 \mu \mathrm{L}$ of the supernatant was aliquoted to a 96-well plate and Malachite Green reagent was used to quantify the release of inorganic phosphate by reading the absorbance at $620 \mathrm{~nm}$. The determination of total SHP-2 protein abundance was performed using the Human/Mouse/Rat Total SHP-2 Kit. In brief, 96-well plates were coated with rat antihuman SHP-2 antibody and then blocked with blocking buffer. After a series of wash steps, $100 \mu \mathrm{L}$ samples involving standard, blank control, or cell lysate was added to each well, respectively, and incubated for $2 \mathrm{~h}$ at room temperature. Wells were aspirated and washed with wash buffer. Diluted biotinylated rat antihuman SHP-2 antibody was added to each well, and plates were incubated for another $2 \mathrm{~h}$. After a series of wash steps, $100 \mu \mathrm{L}$ of the diluted streptavidin-HRP was added and then the plate was incubated for $20 \mathrm{~min}$, protected from light. Stop solution ( $2 \mathrm{~N} \mathrm{H}_{2} \mathrm{SO}_{4}$ ) was added to each well, and absorbance was measured at $450 \mathrm{~nm}$ using a microplate reader (Varioskan Flash, Thermo Fisher Scientific, Waltham, USA).

\section{Immunoprecipitation}

Tosylated superparamagnetic beads (Dynabeads, $2.8 \mu \mathrm{m}$ ) were conjugated with anti-SHP-2 antibody as follows: $200 \mu \mathrm{g}$ antibody with $5 \mathrm{mg}$ dynabeads in $0.1 \mathrm{M}$ borate buffer (pH 8.5) and $1.2 \mathrm{M}\left(\mathrm{NH}_{4}\right)_{2} \mathrm{SO}_{4}$ for $18 \mathrm{~h}$ at $37{ }^{\circ} \mathrm{C}$. Dynabeads conjugated with an irrelevant IgG (Guinea pig $\mathrm{IgG}$ ) or ethanolamine were used as control group. After that, the beads were blocked with excess ethanolamine and then resuspended in pre-cold PBS. The human HepG2 cells were exposed to PFOA $(250 \mu \mathrm{M})$ for $24 \mathrm{~h}$, or with only DMSO as vehicle control. After exposure, all cells were washed twice with ice-cold PBS, and total cellular protein was collected by lysing the cells with RIPA buffer containing a protease inhibitor mixture. The concentration of each protein sample was measured with a BCA Protein Assay Kit. Then, $0.8 \mathrm{mg}$ dynabeads with different surface modification mentioned above were added to cell lysate containing $8 \mathrm{mg}$ protein to immunoprecipitate for $6 \mathrm{~h}$ at $4{ }^{\circ} \mathrm{C}$, respectively. After magnetic separation, dynabeads were washed four times with ice-cold PBS and then pretreated using the ion-pairing extraction method to capture the PFOA on it. 


\section{Molecular docking}

SHP-2 was selected from the Protein Data Bank for constructing the receptor model. All molecular simulation studies on the structural and conformational feature of PFAAs/SHP-2 complexes were performed using Lamarckian genetic algorithm provided by AutoDock 4.2 software. Grid boxes were built around the protein with 60 points cube coverage. A spacing of $0.375 \AA$ between the grid points was used. All other docking parameters were set to defaults, including a population size of 150 , maximum number of 2.5 million evaluations, maximum of 2700 generations, gene mutation rate of 0.02 , crossover rate of 0.8 , and $10 \mathrm{GA}$ runs. Ten docked conformations for each ligand were scored according to a free energy cost function $\left(\Delta G^{*}\right)$, and the lowest-energy conformation was selected as the most likely bioactive conformation.

\section{Results}

\section{Inhibition of SHP-2 activity by PFAAs using an electrochemical enzyme assay}

We first developed an electrochemical biosensor to assay the SHP-2 enzyme activity. The biosensor works on the signal transduction mechanism we reported previously in which tyrosines and phosphorylated tyrosines exhibit differential electrocatalytic current (Qu et al. 2008; Yang et al. 2011). In the biosensor (Fig. 1a), a phosphotyrosine-containing polypeptide was used as the enzyme substrate and was covalently immobilized on the surface of an indium tin oxide (ITO) electrode. Os(bpy) ${ }_{3} \mathrm{Cl}_{2}$ in solution serves as an electrocatalyst. In the presence of SHP-2, phosphortyrosines are converted to tyrosines, which produce a distinctive electrochemical signal. Upon addition of an inhibitor,

$\mathbf{A}$

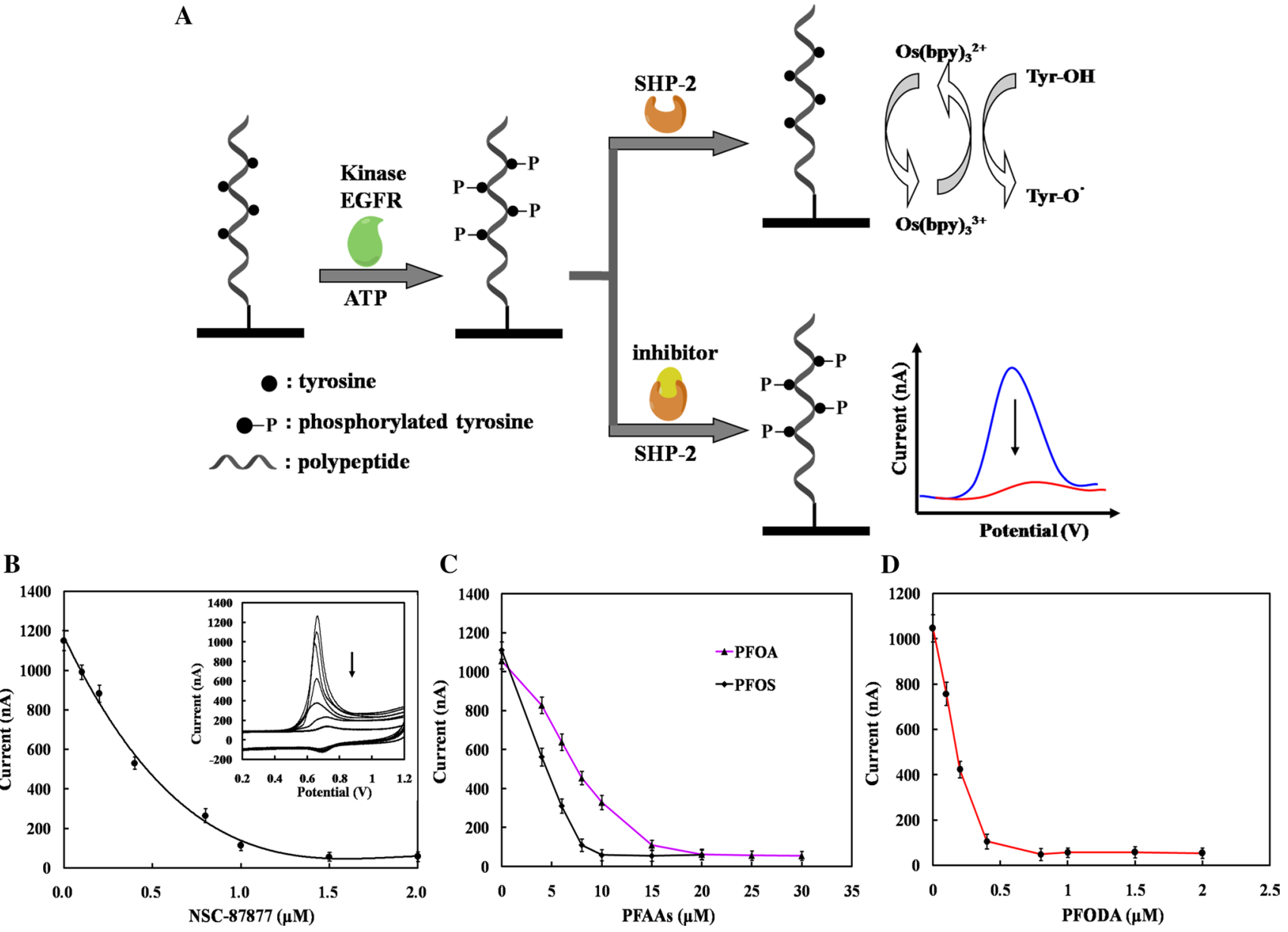

Fig. 1 a Design of an electrochemical biosensor for the assay of SHP-2 enzyme activity and screening of SHP-2 inhibitor. b Dependence of the electrochemical current of the biosensor on the concentration of NSC-87877, a small-molecule inhibitor of SHP-2. Inset cyclic voltammograms of the phosphopolypeptide immobilized on ITO electrode in the presence of various concentrations of NSC-87877.
And dose-effect inhibition curves of PFAAs on SHP-2 enzyme activity, including PFOS, PFOA (c) and PFODA (d). Error bars indicate the standard deviation of three separate measurements $(n=3)$. Electrochemical measurements were taken in $20 \mathrm{mM} \mathrm{PB}$ (pH 7.4) containing $3 \mu \mathrm{M} \mathrm{Os}(\mathrm{bpy})_{3}^{2+}$. The electrode area in contact with the electrolyte was $25 \mathrm{~mm}^{2}$. Voltage scan rate: $30 \mathrm{mVs}^{-1}$ 
Table $1 \mathrm{IC}_{50}, \log K_{\mathrm{ow}}$, and some molecular docking results of PFAAs

\begin{tabular}{lcccll}
\hline PFAAs & Length $(\AA)$ & Log $K_{\text {ow }}$ & $\mathrm{IC}_{50}(\mu \mathrm{M})$ & $\begin{array}{l}\text { Binding energy } \\
(\mathrm{kcal} / \mathrm{M})\end{array}$ & $\begin{array}{l}\text { Hydrogen-bonding } \\
\text { interaction }\end{array}$ \\
\hline PFBA & 6.02 & 2.04 & 20.2 & -5.34 & Lys364, 366, Ser460 \\
PFPA & 6.47 & 2.65 & 16.5 & -5.50 & Lys364, 366, Ser460 \\
PFHxA & 7.94 & 3.25 & 13.1 & -5.78 & Lys364, 366, Ser460 \\
PFHpA & 8.96 & 3.85 & 10.5 & -5.90 & Lys364, 366, Ser460 \\
PFOA & 9.64 & 4.46 & 8.0 & -6.05 & Lys364, 366, Ser460 \\
PFNA & 11.77 & 5.06 & 6.5 & -6.22 & Lys364, 366, Ser460 \\
PFDA & 12.66 & 5.66 & 5.1 & -6.38 & Lys364, 366, Ser460 \\
PFUA & 13.07 & 6.27 & 4.0 & -6.49 & Lys364, 366, Ser460 \\
PFDoDA & 14.08 & 6.87 & 2.8 & -6.55 & Lys364, 366, Ser460 \\
PFTDA & 15.10 & 7.47 & 0.73 & -6.65 & Lys364, 366, Ser460 \\
PFTeDA & 17.15 & 8.08 & 0.51 & -6.75 & Lys364, 366 \\
PFHDA & 19.87 & 9.28 & 0.32 & -6.87 & Lys364, 366 \\
PFODA & 20.98 & 10.49 & 0.18 & -7.09 & Lys364, 366 \\
PFBS & 7.15 & 2.72 & 10.5 & -5.80 & Lys364, 366, Ser460 \\
PFHS & 10.11 & 3.93 & 6.9 & -6.35 & Lys364, 366, Ser460 \\
PFOS & 11.98 & 5.14 & 4.3 & -6.59 & Lys364, 366, Ser460 \\
\hline
\end{tabular}

the signal is reduced due to less conversion of phosphortyrosines. The signal decreased progressively with the concentration of NSC-87877 (a known SHP-2 inhibitor, see Supplementary Material Fig. S1) until it reached a plateau (Fig. 1b). The $\mathrm{IC}_{50}$ and $K_{i}$ values were calculated to be, respectively, $0.52 \pm 0.21$ and $0.37 \pm 0.11 \mu \mathrm{M}$, in accordance with those reported in the literature $\left(\mathrm{IC}_{50}=0.32 \mu \mathrm{M}\right.$, $\left.K_{i}=0.31 \mu \mathrm{M}\right)($ Chen et al. 2006).

After the electrochemical biosensor assay was developed and validated, 16 PFAAs with different carbon chain lengths and acid head groups were screened for their inhibitory effects on SHP-2. As shown in Fig. 1c, d and Supplementary Material Fig. S5, all the PFAAs inhibited SHP-2 activity in a dose-dependent manner. But their inhibition potency differed significantly, as demonstrated by their $\mathrm{IC}_{50}$ values summarized in Table 1 . For the 13 PFCAs, the inhibitory effect increased significantly with their chain length, with PFODA (18 carbons) being the strongest and PFBA (four carbons) the weakest. As for the three PFSAs, the inhibitory effect follows the same pattern, i.e., PFOS > PFHS (six carbons) > PFBS (four carbons, perfluorobutanesulfonic acid). In addition, for the PFSAs and PFCAs containing the same carbon number, PFSAs showed stronger inhibition potency than PFCAs.

\section{Inhibition of SHP-2 activity by PFAAs in HepG2 Cells}

Due to its high expression of SHP-2 enzyme, HepG2 cells were used to further investigate the effect of PFAAs on SHP-2 activity in biological systems. Three PFAAs, PFOA, PFOS, and PFODA, were selected to elucidate the roles of carbon chain length and functional group of PFAAs within cells. HepG2 cells were exposed to one of the PFAAs at non-cytotoxic concentrations for $24 \mathrm{~h}$ (Supplementary Material Fig. S6A-C). Then, SHP-2 was immunoprecipitated from the cell lysates, and its activity was measured by the addition of a synthetic phosphopeptide substrate and quantification of the amount of released phosphate. As shown in Fig. $2 \mathrm{a}-\mathrm{c}$, the three PFAAs were all found to inhibit SHP-2 activity in a concentration-dependent manner. Among them, PFODA reduced SHP-2 activity in HepG2 cells by $50 \%$ at $25 \mu \mathrm{M}$ exposure, and PFOS and PFOA inhibited the activity by 44 and $39 \%$, respectively, at $100 \mu \mathrm{M}$ concentration. Therefore, the inhibition potency follows the order of PFODA > PFOS > PFOA, which is in accordance with the order observed above in the electrochemical biosensor assay and confirms the structural dependence of PFAAs inhibition effect.

Because the enzymatic activity measurement of a protein varies with both total content and specific activity of the protein, we next determined gene expression and protein levels of SHP-2 in HepG2 cells after PFAAs exposure to rule out the first possibility. The results of RT-PCR show that both PFODA and PFOS increased the gene expression of SHP-2 in a concentration-dependent manner, while no effect was observed after PFOA exposure even at the highest concentration (Fig. 2d-f). The SHP-2 protein level in the exposed cells was quantified using Western blot assay (Fig. 2g-i). PFAAs exposure stimulated increase in SHP-2 protein level by 1.19-fold (PFODA), 1.4-fold (PFOS), and 1.3-fold (PFOA), respectively (Fig. 2d-f). The results indicate that SHP-2 inhibition in HepG2 cells by the three PFAAs was due to the loss of enzyme activity, not protein content. 

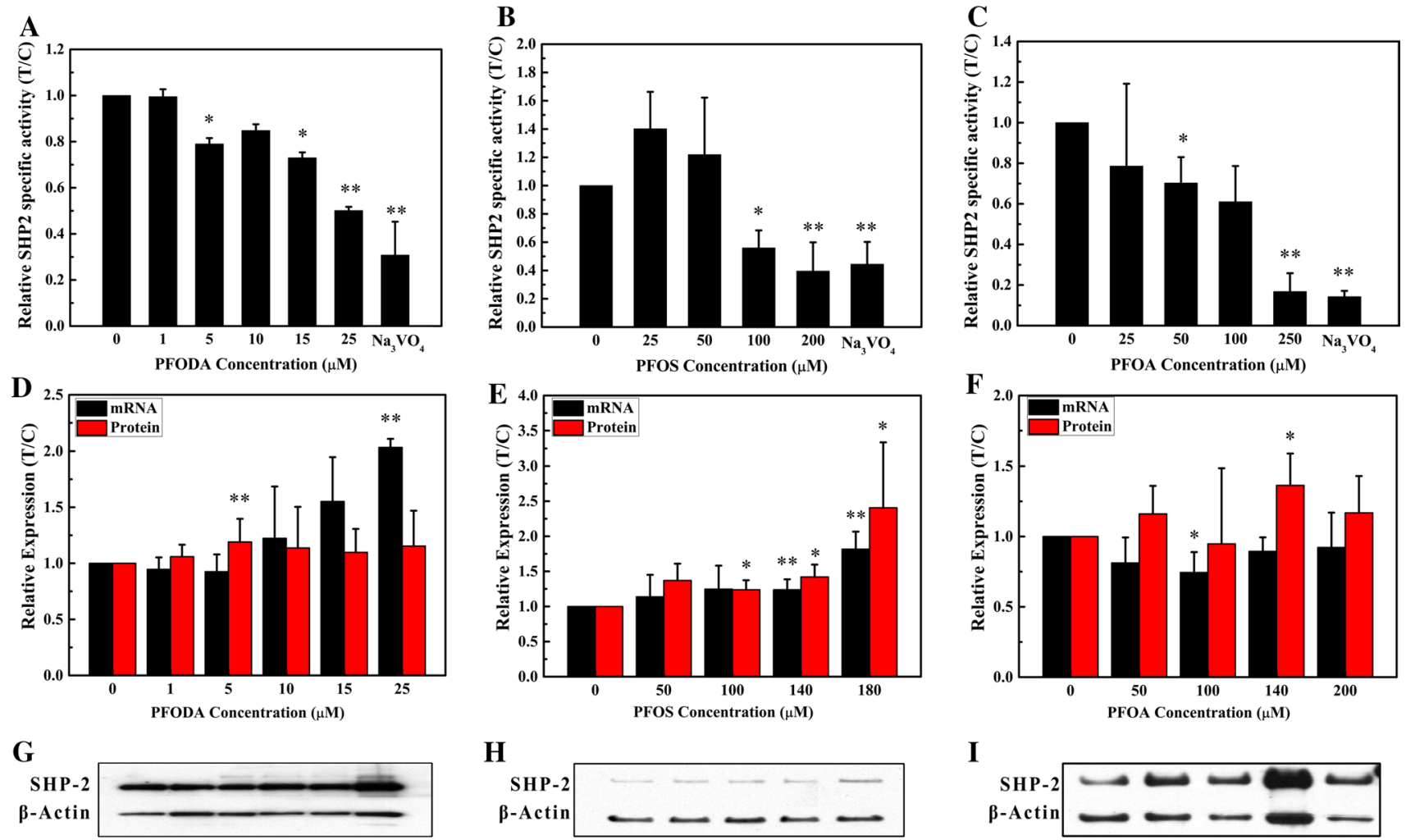

Fig. 2 SHP-2 activity, relative expression of SHP-2 at transcriptional and protein levels, representative images of Western blot analysis on SHP-2 level in HepG2 cells exposed to PFODA (a, d, g), PFOS (b, e, h), and PFOA (c, f, i) for $24 \mathrm{~h}$. HepG2 cells treated with $\mathrm{Na}_{3} \mathrm{VO}_{4}$
$(1 \mu \mathrm{M}, 24 \mathrm{~h})$ were used as positive control. Data are presented as the means of three replicates and normalized against GAPDH (RTPCR) and $\beta$-actin (Western blot). Significance was set as $* p<0.05$; $* * p<0.01$
Fig. 3 Change in paxillin tyrosine phosphorylation level (a) and representative images of Western blot analysis on paxillin tyrosine phosphorylation level (b) in cells exposed to PFODA, PFOS, and PFOA for $24 \mathrm{~h}$. HepG2 cells treated with NSC-87877 (20 $\mu \mathrm{M}, 24 \mathrm{~h})$ were used as positive control. Data represent the mean $\pm \mathrm{SD}$. Significance was set as $* p<0.05$; ** $p<0.01$

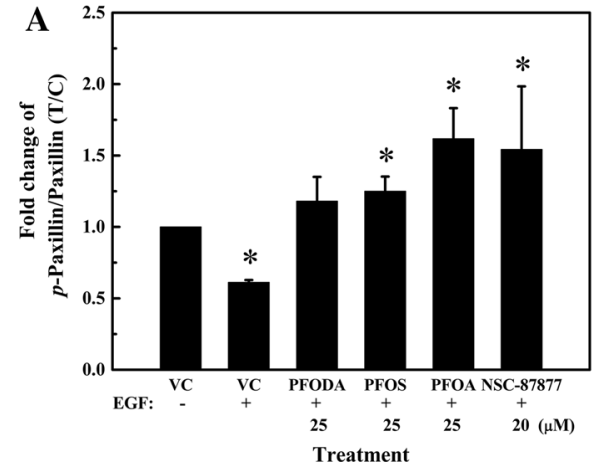

\section{Change in protein tyrosine phosphorylation level after PFAA exposure}

As described in the introduction, protein phosphorylation level in cells is regulated by the coordinated action of protein kinases and protein phosphatases. Since SHP-2 is protein tyrosine phosphatase, its inhibition by PFAAs should lead to an accumulation of phosphotyrosines. We therefore selected paxillin, a physiological substrate of SHP-2, to investigate the effect of PFAA inhibition on protein phosphorylation level in cells. After exposure to one of the three PFAAs, HepG2 cells were lysed and the levels of both paxillin and phosphorylated paxillin were detected by immunoblotting. Compared with the cells treated with culture medium, PFAA-treated cells had a significant increase in the relative content of tyrosine phosphorylated paxillin (Fig. 3a, b). Among the three PFAAs tested, PFOA increased the phosphorylation level as much as NSC-87877 did. 


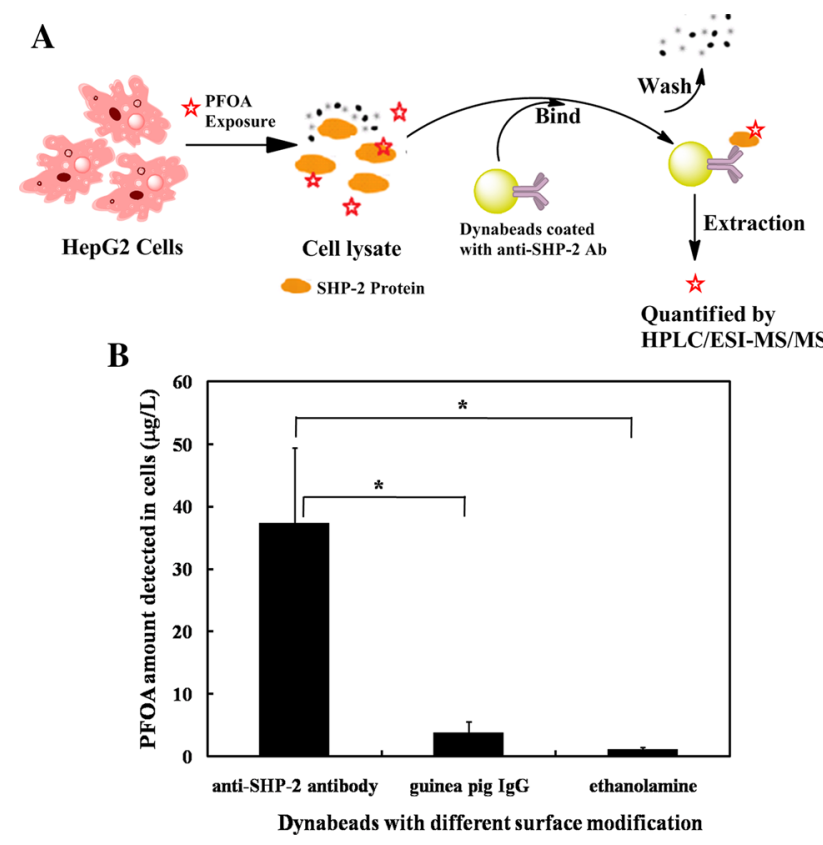

Fig. 4 a Schematic illustration of the capture and quantification of the SHP-2/PFOA complex formed within cells. b PFOA contents detected on magnetic beads with different modifications. *Student's $t$ test, $p<0.05$, compared with guinea pig IgG and ethanolamine modified beads

\section{Detection of PFOA in immunoprecipitated SHP-2 from exposed cells}

Based on the electrochemical biosensor assay results, we hypothesized that the inhibition of SHP-2 activity and increase in protein tyrosine phosphorylation in HepG2 cells after PFAA exposure would be the results of direct binding of PFAA with SHP-2. To prove the hypothesis, HepG2 cells were exposed to $250 \mu \mathrm{M}$ PFOA for $24 \mathrm{~h}$ and then washed extensively to remove the compound that did not enter the cell. Dynabeads coated with anti-SHP-2 antibody were added into the lysate of the exposed cells to capture the protein (Fig. 4a and Supplementary Material Fig. S7). The bead sample was then analyzed for SHP-2 and PFOA content. The amount of SHP-2 captured on the beads was measured to be $0.10 \mathrm{nmol}$ by BCA protein quantification. And the PFOA content detected in the antibody-coated bead sample by HPLC/ESI-MS-MS (Zhou et al. 2014) is $37.33 \mu \mathrm{g} \mathrm{L}^{-1}$ (i.e., $0.09 \mathrm{nmol}$ ), which is approximately 10- and 36-fold higher than the guinea pig IgG-coated bead $\left(3.70 \mu \mathrm{g} \mathrm{L}^{-1}\right)$ and ethanolamine-blocked bead (1.05 $\mu \mathrm{g} \mathrm{L}^{-1}$; Fig. 4b and Supplementary Material Fig. S8). Since both PFOA and SHP-2 were detected on the beads, it can be concluded that PFOA bound directly to SHP-2 in the exposed HepG2 cells. The binding ratio of PFOA to SHP-2 can be estimated to be 0.9 .

\section{Molecular docking simulation of PFAA/SHP-2 complexes}

To better understand the inhibitory effect of PFAAs on SHP-2 activity, the binding interaction of PFAAs with the protein was simulated by molecular docking. Previous X-ray crystal structure shows that the protein comprises two SH2 domains and one catalytic PTP domain. Key conserved features of the PTP catalytic cleft include Cys459, the catalytic nucleophile; Arg465 and the "phosphate-binding cradle" formed by several main-chain amide groups (Hof et al. 1998). We first docked NSC-87877 to SHP-2 using the AutoDock software. Results show that NSC-87877 resides at the PTP catalytic cleft of SHP-2, and its B-ring sulfonic acid group forms hydrogen bonds with the backbone $\mathrm{NH}$ group of Arg465 which is a conserved residue in the PTP signature motif (HCSAGIGRS) located at the base of the PTP catalytic cleft. Due to structural rigidity, the A-ring of NSC-87877 protrudes from the cleft and the sulfonic acid forms hydrogen bonds with the side-chain $\mathrm{NH}_{3}$ group of Lys280 and the side-chain $\mathrm{NH}_{2}$ group of Asn281 (Fig. 5a, b). Our simulation results are in good agreement with those published in the literature (Chen et al. 2006).

By using the same constraints, the 16 PFAAs were docked with SHP-2. Although all the PFAAs were found to bind to the protein at the same cleft as NSC-87877 (Fig. 5c-f and Supplementary Material Fig. S9), they actually exhibited a completely different binding mode. By comparison with NSC-87877, the flexible structure of PFAAs makes them fit to the interior of the cleft. The charged head group of a PFAA interacts with Lys364, Lys366, and Ser460 by hydrogen bonds, and its fluorocarbon tail forms Van der Waals and hydrophobic interactions with the surrounding amino acid residues in the cleft. The binding energy between PFAAs and SHP-2 was calculated and listed in Table 1.

\section{Discussion}

One of the most toxic effects of PFAAs on experimental animals is liver cancer. Unfortunately, the mechanism responsible for the effects of PFAAs on liver has not been clarified. We speculate that some important functional biomolecules might be involved in the mediation of such effects. The inhibition of SHP-2 as a tumor suppressor in liver could in principle lead to the development of tumors in the liver. It also might be a new clue for the toxic and pathogenic factors of PFAAs. In our work, a set of experiments were carried out in an attempt to establish SHP-2 as a potential target of PFAAs in liver cells in their carcinogenic action. 

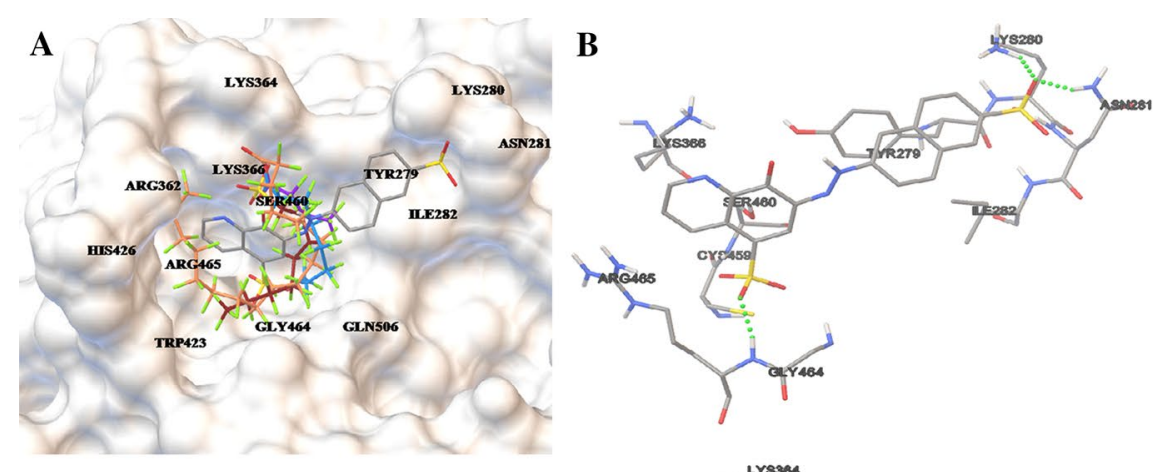

C
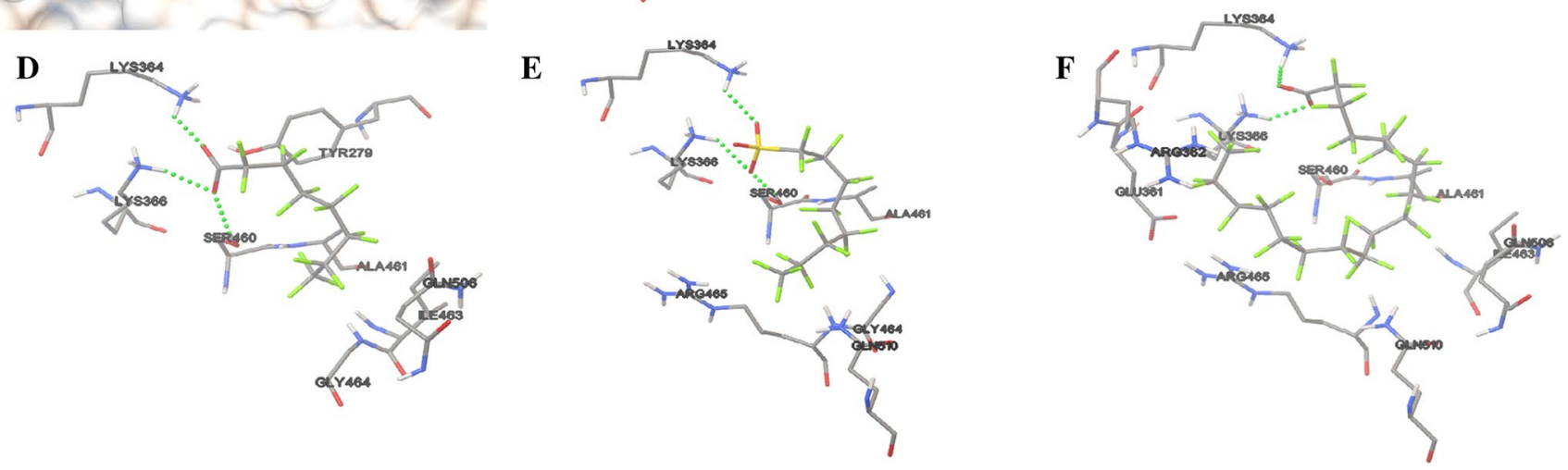

Fig. 5 Molecular model overlay of five compounds bound to the SHP-2 PTP domain, including NSC-87877, PFBA (carbon in purple), PFOA (blue), PFOS (brown), and PFODA (orange; a). The hydrogen bonds formed between SHP-2 and NSC-87877 (b), PFBA (c), PFOA

In the in vitro SHP-2 enzyme activity assay, we found that all the 16 PFAAs inhibited SHP-2 activity for the dephosphorylation of phosphorylated tyrosines in a polypeptide substrate. However, their inhibition potency was strongly dependent on the structures. Among them, the potency of the 13 PFCAs increased progressively with their carbon chain length. The two longest PFCAs, PFHDA and PFODA, were even more potent than NSC-87877, a wellknown SHP-2 inhibitor. A similar trend was also observed for the three PFSAs, although the number of PFSAs under the study was limited. By comparing PFCAs and PFSAs with the same chain length, it is clear that PFSAs are more potent than PFCAs. Molecular docking on the interactions between PFAAs and SHP-2 provided rationales for the difference in their structure-dependent inhibition potency. For the PFCAs, the calculated binding energy of the docked complexes decreased with their carbon chain length, with PFODA being the lowest (Table 1). This trend correlates very well with the experimentally measured inhibition potency $\left(\mathrm{IC}_{50}\right)$ of PFCAs and therefore supports the validity of the molecular docking approach. The agreement in the 1:1 binding mode between the simulated complex and the immunoprecipitation experiment provides further support.

In the docked complexes, all the PFAAs are located in the active center of SHP-2 in such a manner that the entire molecule fits into a binding pocket consisted of amino (d), PFOS (e), and PFODA (f), respectively. For the compounds in $\mathrm{b}-\mathrm{f}$, carbon atoms are colored in gray, oxygen in red, nitrogen in blue, hydrogen in white, sulfur in yellow, and fluorine in green (color figure online)

acids Lys364, Lys366, Ser460, Arg465, Gly464, Arg362, His426, Trp423, Gln506, and Tyr279. But the exact binding geometry is slightly different. For PFCAs from 14 to 18 carbons, there are only two hydrogen bonds between the carboxylate or sulfonate group of the chemical and Lys364, 366 of SHP-2, while for other PFAAs, three hydrogen bonds exist between the carboxylate or sulfonate group of PFAA and the side-chain residues of Lys364, Lys366, and Ser460 of SHP-2. The difference in binding geometry might be that the whole molecule of PFAA moved upward in the cleft with the increase in carbon chain length so as to obtain the optimal matching in size and energy. The calculated binding energy takes into account the hydrophobic, Van der Waal's interactions, and hydrogen-bonding as well as electrostatic interaction. As the fluorocarbon chain of a PFCA gets longer, its hydrophobicity increases, as indicated by its $\log K_{\text {ow }}$. Therefore, long-chain PFCAs should bind stronger to SHP-2 than the short-chain counterparts due to increased hydrophobic and Van der Waal's interactions. In addition, PFAA head group took a leading role in their inhibitory strength. PTP catalytic cleft of SHP-2 is positively charged due to the presence of the "phosphatebinding cradle," and electrostatic interaction is inevitably involved for the combination of PFAA with SHP-2. Sulfonate carries more negative charges and thus is capable of forming stronger electrostatic forces with SHP-2 than 
carboxylate. This may explain why PFSAs are more potent inhibitors than PFCAs even when they possess approximately the same molecular length, $\log K_{\mathrm{ow}}$, and hydrogenbonding mode.

The molecular docking results may also be used to rationalize the structure-dependent inhibition potency observed in the cell-based SHP-2 activity assays, although the situation is more complicated as other processes such as cell permeation may play an important role in the inhibition reaction. Moreover, after cells are exposed to exogenous chemicals such as PFAAs, a variety of biological processes can be perturbed, which might have detrimental effects on SHP-2 activity, either directly or indirectly. To establish that SHP-2 is the direct target of PFAAs in HepG2 cells, we needed to answer the following two questions. First, is the decreased SHP-2 activity in the cell assay due to the loss of SHP-2 activity by PFAA inhibition, or lowering of SHP-2 protein expression in the cell after PFAA exposure? Our experimental results show that neither gene nor protein expression was suppressed by PFAAs. This indicates that the decrease in SHP-2 activity was indeed caused by the inhibition of PFAAs. The other question is does the inhibition occur as a result of PFAAs binding directly to the protein? In the immunoprecipitation experiment, magnetic beads coated with an anti-SHP-2 antibody were used to selectively capture SHP-2 in the cell lysate. During the process, any PFAAs bound to the SHP-2 protein would also be captured by the beads. A significant amount of PFOA was detected by HPLC/ESI-MS-MS in the bead sample. By comparison, minimal PFOA was detected on the uncoated beads, and very low level PFOA was detected on the IgGcoated beads, probably due to some non-specific binding. These results provide unequivocal evidence for the direct binding of PFOA to SHP-2 in HepG2 cells. To the best of our knowledge, this is the first time a complex between an environmental chemical and a protein was detected in cells.

Since SHP-2 participates in the regulation of protein tyrosine phosphorylation level by catalyzing the removal of phosphate, inhibition of SHP-2 activity should lead to an increase in phosphotyrosines of its substrate. We chose paxillin as a protein substrate of SHP-2 to evaluate the effect of SHP-2 inhibition in HepG2 cells. As expected, all the three PFAAs induced significant increase in phosphorylated paxillin in HepG2 cells. Paxillin is a scaffold protein located to the intracellular surface of the cell membrane. It interacts with a wide variety of structural and regulatory proteins through its multiple binding domains, many of which are regulated by tyrosine phosphorylation. The state of paxillin phosphorylation plays a central role in cell adhesion, migration, and metastasis, and it is regulated in turn by SHP-2 and other phosphatases (Deakin and Turner 2008). The evidence provided in our study possibly explained the association between PFAA exposure and its carcinogenicity. It remains to be seen whether SHP-2 inhibition by PFAAs would have any impacts on cell motility.

\section{Conclusions}

In summary, using a combined experimental approach including biosensor-based enzyme activity assay, cellbased assays, immunoprecipitation, and molecular docking simulation, we have established protein tyrosine phosphatase SHP-2 as a new target of PFAAs in cells. Upon cell exposure, PFAAs enter the cell, bind directly to SHP-2, and inhibit its tyrosine dephosphorylation reaction, and thereby increase the level of phosphorylated tyrosine residues of paxillin. Further studies are needed to investigate the effects of SHP-2 inhibition on cell motility and its possible link to the toxic effects observed on experimental animals.

Acknowledgments This work was supported by the Chinese Academy of Sciences (XDB14040100) and National Natural Science Foundation of China (Nos. 21321004, 21577163, 21377142, 21377145, 21477146, 21407168, 91543203).

\section{Compliance with ethical standards}

Conflict of interest None of the authors have any conflict of interest to declare.

\section{References}

Bard-Chapeau EA, Li SW, Ding J, Zhang SS, Zhu HH, Princen F, Fang DD, Han T, Bailly-Maitre B, Poli V (2011) Ptpn11/Shp2 acts as a tumor suppressor in hepatocellular carcinogenesis. Cancer Cell 19(5):629-639

Barry V, Winquist A, Steenland K (2013) Perfluorooctanoic acid (PFOA) exposures and incident cancers among adults living near a chemical plant. Environ Health Perspect 121(11-12):1313-1318

Benninghoff AD, Bisson WH, Koch DC, Ehresman DJ, Kolluri SK, William DE (2011) Estrogen-like activity of perfluoroalkyl acids in vivo and interaction with human and rainbow trout estrogen receptors in vitro. Toxicol Sci 120(1):42-58

Butterworth S, Overduin M, Barr AJ (2014) Targeting protein tyrosine phosphatase SHP2 for therapeutic intervention. Future Med Chem 6(12):1423-1437

Chang ET, Adami HO, Boffetta P, Cole P, Starr TB, Mandel JS (2014) A critical review of perfluorooctanoate and perfluorooctane sulfonate exposure and cancer risk in humans. Crit Rev Toxicol 44(1):1-81

Chen LW, Sung SS, Yip MLR, Lawrence HR, Ren Y, Guida WC, Sebti SM, Lawrence NJ, Wu J (2006) Discovery of a novel Shp2 protein tyrosine phosphatase inhibitor. Mol Pharmacol 70(2):562-570

Conder JM, Hoke RA, de Wolf W, Russell MH, Buck RC (2008) Are PFCAs bioaccumulative? A critical review and comparison with regulatory lipophilic compounds. Environ Sci Technol 42(4):995-1003

Deakin NO, Turner CE (2008) Paxillin comes of age. J Cell Sci 121(15):2435-2444 
Deb TB, Wong L, Salomon DS, Zhou GC, Dixon JE, Gutkind JS, Thompson SA, Johnson GR (1998) A common requirement for the catalytic activity and both SH2 domains of SHP-2 in mitogen-activated protein (MAP) kinase activation by the ErbB family of receptors-a specific role for SHP-2 in MAP, but not c-jun amino-terminal kinase activation. J Biol Chem 273(27):16643-16646

DeWitt J, Shnyra A, Badr M, Loveless S, Hoban D, Frame S, Cunard R, Anderson S, Meade BJ, Peden-Adams M (2009) Immunotoxicity of perfluorooctanoic acid and perfluorooctane sulfonate and the role of peroxisome proliferator-activated receptor alpha. Crit Rev Toxicol 39(1):76-94

Eriksen KT, Sorensen M, McLaughlin JK, Lipworth L, Tjonneland A, Overvad K, Raaschou-Nielsen O (2009) Perfluorooctanoate and perfluorooctane sulfonate plasma levels and risk of cancer in thegeneral Danish population. J Natl Cancer Inst 101(8):605-609

Florentin A, Deblonde T, Diguio N, Hautemaniere A, Hartemann P (2011) Impacts of two perfluorinated compounds (PFOS and PFOA) on human hepatoma cells: cytotoxicity but no genotoxicity? Int J Hyg Environ Health 214(6):493-499

Giesy JP, Kannan K (2002) Perfluorochemical surfactants in the environment. Environ Sci Technol 36(7):146A-152A

Griffith FD, Long JE (1980) Animal toxicity studies with ammonium perfluorooctanoate. Am Ind Hyg Assoc J 41(8):576-583

Hamann TW, Gstrein F, Brunschwig BS, Lewis NS (2005) Measurement of the free-energy dependence of interfacial charge-transfer rate constants using $\mathrm{ZnO} / \mathrm{H}_{2} \mathrm{O}$ semiconductor/liquid contacts. J Am Chem Soc 127(21):7815-7824

Hof P, Pluskey S, Dhe-paganon S, Eck MJ, Shoelson SE (1998) Crystal structure of the tyrosine phosphatase SHP-2. Cell 92(4):441-450

Humblet O, Diaz-Ramirez LG, Balmes JR, Pinney SM, Hiatt RA (2014) Perfluoroalkyl chemicals and asthma among children 12-19 years of age: NHANES (1999-2008). Environ Health Perspect 122(10):1129-1133

Jiang CY, Hu FK, Tai YH, Du JL, Mao BB, Yuan ZQ, Wang Y, Wei LX (2012) The tumor suppressor role of Src homology phosphotyrosine phosphatase 2 in hepatocellular carcinoma. J Cancer Res Clin Oncol 138(4):637-646

Kato K, Wong LY, Jia LT, Kuklenyik Z, Calafat AM (2011) Trends in exposure to polyfluoroalkyl chemicals in the U.S. population: 1999-2008. Environ Sci Technol 45(19):8037-8045

Kristensen SL, Ramlau-Hansen CH, Ernst E, Olsen SF, Bonde JP, Vested A, Halldorsson TI, Becher G, Haug LS, Toft G (2013) Long-term effects of prenatal exposure to perfluoroalkyl substances on female reproduction. Hum Reprod 28(12):3337-3348

Kudo N, Suzuki-Nakajima E, Mitsumoto A, Kawashima Y (2006) Responses of the liver to perfluorinated fatty acids with different carbon chain length in male and female mice: in relation to induction of hepatomegaly, peroxisomal beta-oxidation and microsomal 1-acylglycerophosphocholine acyltransferase. Biol Pharm Bull 29(9):1952-1957

Li S, Hsu DDF, Wang H, Feng GS (2012) Dual faces of SH2-containing protein-tyrosine phosphatase Shp2/PTPN11 in tumorigenesis. Front Med 6(3):275-279

Liu DP, Kong GP, Chen QC, Wang GH, Li J, Xu Y, Lin T, Tian YP, Zhang XK, Yao XS (2011) Fatty acids as natural specific inhibitors of the proto-oncogenic protein Shp2. Bioorg Med Chem Lett 21(22):6833-6837

Mohi MG, Neel BG (2007) The role of SHP-2 (PTPN11) in cancer. Curr Opin Genet Dev 17(1):23-30

Muller PJ, Rigbolt KTG, Paterok D, Sobota RM (2013) Protein tyrosine phosphatase SHP-2/PTPN11 mistargeting as a consequence of SH2-domain point mutations associated with Noonan syndrome and leukemia. J Proteom 84:132-147
Ng CA, Hungerbuhler K (2014) Bioaccumulation of perfluorinated alkyl acids: observations and models. Environ Sci Technol 48(9):4637-4648

Olsen GW, Burris JM, Ehresman DJ, Froehlich JW, Seacat AM, Butenhoff JL, Zobel LR (2007) Half-life of serum elimination of perfluorooctane sulfonate, perfluorohexane sulfonate, and perfluorooctanoate in retired fluorochemical production workers. Environ Health Perspect 115(9):1298-1305

Perez F, Nadal M, Navarro-Ortega A, Fabrega F, Domingo JL, Barcelo D, Farre M (2013) Accumulation of perfluoroalkyl substances in human tissues. Environ Int 59:354-362

Qu N, Wan B, Guo LH (2008) Label-free electrochemical differentiation of phosphorylated and non-phosphorylated peptide by electro-catalyzed tyrosine oxidation. Analyst 133(9):1246-1249

Ren XM, Zhang YF, Guo LH, Qin ZF, Lv QY, Zhang LY (2015) Structure-activity relations in binding of perfluoroalkyl compounds to human thyroid hormone T3 receptor. Arch Toxicol 89(2):233-242

Sato I, Kawamoto K, Nishikawa Y, Tsuda S, Yoshida M, Yaegashi K, Saito N, Liu W, Jin YH (2009) Neurotoxicity of perfluorooctane sulfonate (PFOS) in rats and mice after single oral exposure. J Toxicol Sci 34(5):569-574

Simpson C, Winquist A, Lally C, Steenland K (2013) Relation between perfluorooctanoic acid exposure and strokes in a large cohort living near a chemical plant. Environ Res 127:22-28

Tartaglia M, Mehler EL, Goldberg R, Zampino G, Brunner HG, Kremer H, van der Burgt I, Crosby AH, Ion A, Jeffery S (2001) Mutations in PTPN11, encoding the protein tyrosine phosphatase SHP-2, cause Noonan syndrome. Nat Genet 29(4):465-468

Tartaglia M, Niemeyer CM, Shannon KM, Loh ML (2004) SHP-2 and myeloid malignancies. Curr Opin Hematol 11(1):44-50

Toft G, Jonsson BAG, Lindh CH, Giwercman A, Spano M, Heederik D, Lenters V, Vermeulen R, Rylander L, Pedersen HS (2012) Exposure to perfluorinated compounds and human semen quality in arctic and European populations. Hum Reprod 27(8):2532-2540

VandenHeuvel JP, Thompson JT, Frame SR, Gillies PJ (2006) Differential activation of nuclear receptors by perfluorinated fatty acid analogs and natural fatty acids: a comparison of human, mouse, and rat peroxisome proliferator-activated receptor-alpha, -beta, and -gamma, liver $X$ receptor-beta, and retinoid $X$ receptoralpha. Toxicol Sci 92(2):476-489

Vieira VM, Hoffman K, Shin HM, Weinberg JM, Webster TF, Fletcher T (2013) Perfluorooctanoic acid exposure and cancer outcomes in a contaminated community: a geographic analysis. Environ Health Perspect 121(3):318-323

Wang SF, Lv QY, Yang Y, Guo LH, Wan B, Zhao LX (2014a) Cellular target recognition of perfluoroalkyl acids: in vitro evaluation of inhibitory effects on lysine decarboxylase. Sci Total Environ 496:381-388

Wang Y, Rogan WJ, Chen PC, Lien GW, Chen HY, Tseng YC, Longnecker MP, Wang SL (2014b) Association between maternal serum perfluoroalkyl substances during pregnancy and maternal and cord thyroid hormones: Taiwan maternal and infant cohort study. Environ Health Perspect 122(5):529-534

Wang F, Liu W, Jin YH, Wang FQ, Ma JS (2015) Prenatal and neonatal exposure to perfluorooctane sulfonic acid results in aberrant changes in miRNA expression profile and levels in developing rat livers. Environ Toxicol 30(6):712-723

White SS, Fentona SE, Hines EP (2011a) Endocrine disrupting properties of perfluorooctanoic acid. J Steroid Biochem Mol Biol 127(1-2):16-26

White SS, Stanko JP, Kato K, Calafat AM, Hines EP, Fenton SE (2011b) Gestational and chronic low-dose PFOA exposures and mammary gland growth and differentiation in three generations of CD-1 mice. Environ Health Perspect 119(8):1070-1076 
Wolf CJ, Schmid JE, Lau C, Abbott BD (2012) Activation of mouse and human peroxisome proliferator-activated receptor-alpha (PPAR $\alpha$ ) by perfluoroalkyl acids (PFAAs): further investigation of C4-C12 compounds. Reprod Toxicol 33(4):546-551

Yang Q, Xie Y, Alexson SHE, Nelson BD, DePierre JW (2002) Involvement of the peroxisome proliferator-activated receptor alpha in the immunomodulation caused by peroxisome proliferators in mice. Biochem Pharmacol 63(10):1893-1900

Yang Y, Guo LH, Qu N, Wei MY, Zhao LX, Wan B (2011) Label-free electrochemical measurement of protein tyrosine kinase activity and inhibition based on electro-catalyzed tyrosine signaling. Biosens Bioelectron 28(1):284-290

Yu B, Liu W, Yu WM, Loh ML, Alter S, Guvench O, MacKerell AD, Tang LD, Qu CK (2013) Targeting protein tyrosine phosphatase SHP2 for the treatment of PTPN11-associated malignancies. Mol Cancer Ther 12(9):1738-1748
Zhang H, Shi Z, Liu Y, Wei Y, Dai J (2008) Lipid homeostasis and oxidative stress in the liver of male rats exposed to perfluorododecanoic acid. Toxicol Appl Pharm 227(1):16-25

Zhang LY, Ren XM, Guo LH (2013a) Structure-based investigation on the interaction of perfluorinated compounds with human liver fatty acid binding protein. Environ Sci Technol 47(19):11293-11301

Zhang T, Guo WJ, Yang Y, Liu W, Guo LL, Gu YH, Shu YQ, Wang L, Wu XF, Hua ZC (2013b) Loss of SHP-2 activity in CD4(+) $\mathrm{T}$ cells promotes melanoma progression and metastasis. Sci Rep 3:1-10

Zhou Z, Shi YL, Vestergren R, Wang T, Liang Y, Cai YQ (2014) Highly elevated serum concentrations of perfluoroalkyl substances in fishery employees from Tangxun Lake, China. Environ Sci Technol 48(7):3864-3874 